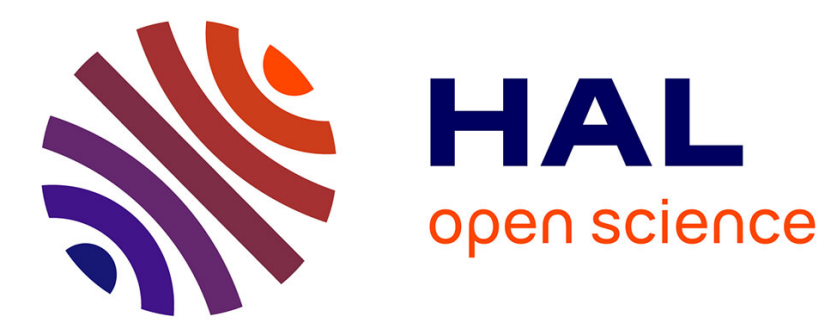

\title{
La traçabilité comme technique de gouvernement des hommes et des choses
}

Didier Torny

\section{To cite this version:}

Didier Torny. La traçabilité comme technique de gouvernement des hommes et des choses. Politix, 1998, Politiques du risque, 44, pp.51-75. 10.3406/polix.1998.1761 . halshs-01249086

\section{HAL Id: halshs-01249086 \\ https://shs.hal.science/halshs-01249086}

Submitted on 30 Dec 2015

HAL is a multi-disciplinary open access archive for the deposit and dissemination of scientific research documents, whether they are published or not. The documents may come from teaching and research institutions in France or abroad, or from public or private research centers.
L'archive ouverte pluridisciplinaire HAL, est destinée au dépôt et à la diffusion de documents scientifiques de niveau recherche, publiés ou non, émanant des établissements d'enseignement et de recherche français ou étrangers, des laboratoires publics ou privés.

\section{(c)(1)}

Distributed under a Creative Commons Attribution| 4.0 International License 


\section{la traçabilité comme technique de gouvernement des hommes et des choses}

Résumé :

Didier Torny

Groupe de Sociologie Politique et Morale (GSPM)

Face à l'émergence de nouvelles menaces sanitaires, les politiques traditionnelles de protection et d'isolement du territoire (quarantaine) se révèlent insuffisantes. Pour répondre à des dangers qui sont intimement liés à la circulation croissante des biens et des personnes, on assiste à la mise en place progressive de la traçabilité comme nouveau modèle de gouvernementabilité. Cet article décrit les contraintes qui s'exercent en pratique sur la mise en place de cette traçabilité à partir des dossiers des maladies à prions ("vache folle", Maladie de Creutzfeldt-Jakob), de la transfusion sanguine et des Organismes Génétiquement Modifiés (OGM).

Tracability as technique of governance of people and goods

Summary :

The traditional politics of territorial protection and isolation (quarantine) seem inefficient to face new emerging sanitary threats. To cope with dangers intertwined with growing movements of people and goods, we are witnessing the ongoing settlement of tracability as technique of governance. This article focuses on the practical constraints to build this tracability through the fields of prion diseases (" mad cow disease", Creutzfeldt-Jakob disease), blood transfusion and Genetically Engineered Foods.

\footnotetext{
“Il entre dans les corps, il infeste. Son pouvoir infectieux se mesure à sa capacité de s'adapter à un ou plusieurs hôtes. Cette capacité fluctue, et sa virulence varie, et sa production de substances toxiques. Elles sommeillent, s'exaltent, s'exaspèrent, peuvent se perdre longuement. "Michel Serres, Le Parasite.
}

Cet article traite des difficultés auxquelles sont aujourd'hui confrontés les pouvoirs publics, en France comme dans d'autres pays occidentaux, pour tenter de maintenir ou d'améliorer la sécurité sanitaire au sein de vastes réseaux de production et de distribution économique dont 
l'extension dépasse largement leur territoire de compétence ${ }^{1}$. Il s'agit là d'un problème de gouvernementabilité assez nouveau. Longtemps, en effet, les Etats ont fait face aux menaces sanitaires qui pesaient sur leurs populations de façon territorialisée, en utilisant le système du quadrillage, de l'isolement et de la mise en quarantaine ${ }^{2}$. Le développement des échanges et des réseaux de communication rend de plus en plus inefficaces et difficiles à mettre en œuvre ces systèmes de bouclage territorial : d'une part, les déplacements s'intensifiant, les exigences de libre circulation des personnes et des biens deviennent de plus en plus incompatibles avec l'arrêt brutal des circulations qu'exige dans le modèle de la quarantaine l'impératif de sécurité ; d'autre part, la multiplication des déplacements d'humains et de non-humains entre les nations rend de plus en plus aléatoire le contrôle et la sanctuarisation des frontières.

Depuis quelques années, on assiste à l'émergence d'un autre modèle de contrôle des populations et des choses, complémentaire au modèle de la contention et de la clôture territoriale. Ce modèle s'appuie sur de nouveaux outils, pratiques et dispositifs de gouvernement rassemblés généralement autour de la notion de "traçabilité". En suivant M.-A. Hermitte, il est possible de distinguer au moins trois usages du mot "traçabilité" ${ }^{3}$. En premier lieu, la traçabilité peut servir à la lutte contre les illicéites d'usage. On entend par là le fait qu'un certain nombre de produits en vente libre peuvent donner lieu à des usages illicites (par exemple, les produits indispensables à la fabrication de certaines drogues, appelés "précurseurs de la drogue"). Une traçabilité de ces produits permettrait de suspecter et de repérer la production de stupéfiants ou, au niveau du consommateur, le changement de composition de produits. Dans le domaine des maladies à prions ("vache folle", Maladie de Creutzfeldt-Jakob), on peut évoquer de la même façon l'exemple des farines animales. La fabrication de ces farines est légale mais leur utilisation comme nourriture pour les bovins et plus généralement, les ruminants est aujourd'hui interdite. Cette interdiction n'a pas empêché l'utilisation volontaire ou involontaire de ces farines, censées n'être plus destinées qu'aux porcs et aux volailles, pour des bovins ${ }^{4}$. En second lieu, la traçabilité peut être utilisée comme outil de sécurité a posteriori . Il s'agit alors “de retrouver les objets dangereux une fois qu'ils ont été commercialisés"s. Si les retrouver est primordial, c'est évidemment en vue d'agir sur ces produits (expertise, consigne, destruction...). Enfin, la traçabilité peut servir d'outil de prévention des risques. Dans ce cas, elle est destinée à rendre "illicites des circulations non

\footnotetext{
${ }^{1}$ Je remercie Cyril Lemieux pour ses remarques et son travail sur une première version de ce texte.

${ }^{2}$. Foucault (M.), Surveiller et Punir, Paris, Gallimard, 1975.

3. Hermitte (M.-A.), "L'illicite dans le commerce international des marchandises", in "L'illicite dans le commerce International", Travaux du CREDIMI, vol. 16, 1996.

${ }^{4}$. On pourrait encore citer le cas des hormones de croissance bovine, soupçonnées d'être frauduleusement utilisés par les sportifs et surtout les culturistes.

${ }^{5}$. Hermitte M.-A., op.cit.
} 
maîtrisables". Ce qui passe en particulier par la mise en place des liste positives de produits, comme nous le verrons entre autres dans le cas des dispositifs médicaux implantables.

Nous nous intéresserons ici principalement aux deux derniers usages de la traçabilité. La question que nous nous posons est de savoir à quels types de transformations dans l'ordre socio-politique tend à correspondre la mise en place progressive de la traçabilité comme nouveau modèle de gouvernementabilité ${ }^{6}$. Quels types d'intervention sont attendues de l'Etat dans ce modèle ? Quelles modalités de prise de décision y sont promues ? Quel type de compétences citoyennes y sont réclamées ? Un examen de ces différentes dimensions peut permettre de cerner le genre de difficultés que rencontrent les promoteurs du nouveau modèle pour satisfaire deux contraintes tendanciellement antagonistes : d'une part, la garantie de sécurité due par l'Etat aux citoyens ; d'autre part, le maintien d'un niveau élevé d'échanges et de déplacements, compatibles avec les principes d'organisation libérale de l'économie mondiale. Nos exemples porteront notamment sur des cas récents liés aux prions, à la transfusion sanguine ou encore aux Organismes Génétiquement Modifiés (OGM) ${ }^{7}$.

\section{Les limites du modèle de la quarantaine face à l'extension des parasites}

Le fait que l'activité humaine génère par elle-même des dangers inédits est fort connu et les règles de droit en ont largement attribué la responsabilité au producteur de l'activité dès la fin du XIXème siècle. Plus tard, dans des domaines jugés particulièrement risqués ou liés à la sécurité de l'Etat, comme l'industrie aéronautique, nucléaire et de l'armement, se sont développées les premières formes de contrôle qui se rapprochent de la traçabilité actuelle ${ }^{8}$. Ces formes de suivi, comme nous allons le voir, sont appelées à se développer aujourd'hui en raison de ce qui fait la caractéristique majeure de certaines menaces contemporaines : elles réunissent la capacité de prolifération des "maladies d'hier", épidémies aériennes ou de contact (peste, choléra, grippe) liées au vivant, et que leur mode de prolifération suit

\footnotetext{
${ }^{6}$. Nous reviendrons sur la capacité d'extension de ce modèle à bien d'autres domaines que la gestion des risques collectifs : le domaine répressif (comme nous l'avons évoqué à propos des stupéfiants), le domaine social (lorsque la traçabilité vise à reconstituer les conditions sociales dans lesquelles certains marchandises et services importés sont produits dans les pays d'origine : travail des enfants, sous-rémunération des producteurs, etc.) ou encore, le domaine commercial (à travers la mise en place de labels et d'appellations d'origine).

${ }^{7}$. Cet article est largement issu d'un travail collectif sur les "lanceurs d'alerte" : Chateauraynaud (F.), Hélou (C.), Lemieux (C.) et Torny (D.), Alertes et prophéties .Les risques collectifs entre vigilance, controverse et critique, Rapport du programme Risques Collectifs et Situations de Crise du CNRS, décembre 1997, 2 tomes.

${ }^{8}$. Wright (W.), Building the future, London, Oxford University Press, 1982.
}

${ }^{9}$ Herzlich -(C.), Pierret (J.), Malades d'hier, malades d'aujourd'hui, Paris, Payot, 1984. 
étroitement les réseaux socio-techniques mis en place par les humains, ce qui en fait de véritables parasites. C'est cette superposition de propriétés qui transforme l'utilisation de mesures de protections classiques en remèdes inefficaces. ${ }^{10}$

Soit par exemple le cas de ces deux cent soixante-dix personnes qui ont reçu en Irlande du sang provenant d'un donneur britannique qui s'est révélé par la suite être porteur de la nouvelle variante de la maladie de Creutzfeldt-Jakob (MCJ), un fléau qui a déjà tué vingtdeux personnes en Grande-Bretagne. Les produits sanguins auraient été transfusés dans neuf hôpitaux irlandais avant d'être détruits le 26 novembre 1997, après le décès du donneur ${ }^{11}$. Dans une circonstance comme celle-ci, il apparaît que le mode de fabrication des produits dérivés du sang (un mode industriel) et leur mode de diffusion (un mode commercial) font quasi immédiatement d'une alerte au départ locale un problème à gérer au niveau mondial. $\mathrm{A}$ l'occasion de cette alerte, le fabricant du produit, le laboratoire britannique Nycomed Amersham, a en effet révélé que le lot de produits suspects avait pu avoir été vendu dans cinquante-deux pays au total, où la société compte des clients réguliers de cette substance! Les responsables de ce laboratoire ont ainsi estimé avoir vendu au moins dix mille unités du produit incriminé entre juin et septembre ou octobre de l'année $1997^{12}$. Cet exemple souligne comment, dans un monde où les procédés industriels se sont généralisés (ici notamment à travers le "poolage" des dons) et où les échanges de toute nature se sont intensifiés à un point qui n'avait jamais été atteint, les produits issus du sang d'un seul donneur peuvent, en quelques mois, se trouver disséminés sur toute la planète et être investis dans des applications extrêmement diverses (des immunoglobulines antitétaniques aux utilisations en injection par des radiologues, comme c'est le cas ici). Les réseaux de production et de circulation créent ainsi, à leur insu, des milliers de liens entre des personnes auparavant séparées, liens potentiellement propices à de nouvelles formes de menaces sanitaires.

Dans Surveiller et Punir, M. Foucault a décrit les dispositifs disciplinaires et punitifs qui visaient (et visent toujours) à lutter contre les dangers provenant directement des humains. Il

\footnotetext{
${ }^{10}$ Nous suivons M. Serres dans son utilisation sémantique étendue du parasite : il ne s'agit pas ici de se limiter à la parasitologie comme simple discipline biologique. M. Serres bâtit une cosmologie où la relation parasitaire est la relation sociale primaire, le contrat n'étant que second. En ce sens, le parasite n'est pas nécessairement dangereux ou même défavorable à l'hôte ; de même les menaces que nous traitons relèvent le plus souvent d'une logique de faibles doses, la question de leur dangerosité étant souvent seconde par rapport à celle de la mise en évidence de leur présence parasitaire. Serres (M.), Le parasite, Paris, Pluriel, 1980.

11. Selon Le Figaro du 15 décembre 1997.

${ }^{12}$. Ils adresseront des mises en garde à tous leurs clients, tout en assurant que le retrait des lots suspects doit être compris comme une "mesure de précaution" car, rappellent-ils, il n'est pas établi que la nouvelle variante de la MCJ soit transmissible par le sang. Selon l'AFP du 15 décembre 1997.
} 
soulignait le rôle central du quadrillage de l'espace et associait le fonctionnement des hôpitaux à ce modèle de mise en isolement :

Les aménagements de la surveillance fiscale et économique précédent les techniques de l'observation médicale : localisation des médicaments dans des coffres fermés, registre de leur utilisation ; un peu plus tard, on met au point un système pour vérifier le nombre réel des malade, leurs allées et venues, on les contraint à rester dans leurs salles; à chaque lit est attaché le nom de qui s'y trouve ; tout individu soigné est porté sur un registre que le médecin doit consulter pendant la visite; plus tard viendront l'isolement des contagieux, les lits séparés ${ }^{13}$.

Ce type de mesures demeure évidemment valide par rapport à la plupart des pathologies infectieuses, tant humaines qu'animales. Cependant, la circulation intensive des personnes modifie le quadrillage de l'espace, qui n'est plus fixe mais suit désormais les déplacement des agents à surveiller, que ce soient des hommes ou des produits. Considérons par exemple les mesures prises suite à la découverte d'un cas de méningite, maladie rare mais à la mortalité foudroyante chez les enfants. Un petit garçon, originaire d'Indre-et-Loire, avait séjourné du 20 au 30 août 1998 dans une famille d'accueil à Pomas, entre Carcassonne et Limoux. Il avait été hospitalisé pour une méningite dès son retour de vacances, à Tours. Prévenus par la DDASS d'Indre-et-Loire, les services sanitaires de l'Aude ont fait mettre en oeuvre une chimioprophylaxie par antibiotiques pour toutes les personnes ayant été directement en contact avec l'enfant. Il s'agit d'un traitement préventif (un responsable de la DDASS souligne que "le germe est très sensible" et "la contagiosité très rare"). Là où un découpage territorial aurait été autrefois envisageable, la multiplication des déplacements rend impossible le découpage entre une zone saine et une zone potentiellement contaminée. Dans un cas comme celui-ci, les modèles de "ligne Maginot" ou de quarantaine, bien adaptés à des alertes à faible extension territoriale, risquent d'être insuffisants. La traçabilité, comme nous allons le voir, tente de réadapter ces modèles, mais autour de nouvelles topologies dont la caractéristique principale est l'absence de continuité territoriale.

Pour répondre à la menace, on passe ainsi d'une division claire de l'espace territorial à la reconstitution d'un réseau de personnes et d'objets ayant été au contact de la menace supposée ou réelle. Dans cet exemple d'alerte à la méningite, la reconstitution est relativement simple, du fait, d'une part, que seuls les humains sont concernés, d'autre part que le temps d'incubation de la pathologie est très court. Comme on va le voir, certains parasites potentiellement vecteurs de risques sanitaires ont une capacité à demeurer longtemps silencieux et à se fixer également sur d'autres hôtes que les humains, ce qui rend d'autant plus complexe la reconstitution du réseau d'entités ayant été au contact de la menace.

\footnotetext{
${ }^{13}$. Foucault (M.), Surveiller et Punir, op. cit., p.169.
} 
Une préoccupation montante : reconstituer les réseaux

Regrouper dans des catégories adéquates, dénombrer, puis isoler a longtemps été la tâche essentielle des acteurs chargés de la santé publique. Pour faire face aux nouveaux modes de prolifération des menaces sanitaires, cette préoccupation tend désormais à être supplantée par une autre : il s'agit de reconstituer des réseaux et des parcours "individualisés" - ainsi dans notre exemple d'alerte à la méningite. Il s'agit là d'une opération familière au sociologue des sciences et des techniques. "L'école de Paris" de la nouvelle histoire des sciences s'est en effet développée autour de la notion d'“acteur-réseau" pour décrire le développement et l'extension de dispositifs socio-techniques. Pour B. Latour, l'extension des réseaux sociotechniques est l'essence même de l'apparente différence entre monde moderne et prémoderne, différence qui est pour lui quantitative et non qualitative ${ }^{14}$. Les principales productions de cette école analysent l'activité d'innovateurs à travers la description de pratiques liées à un réseau d'humains et de non humains. Le réseau, à la fois technique et social, est donc ici un opérateur descriptif neutre qui rend compte de la création du fonctionnement ou de l'arrêt d'activités ${ }^{15}$. Cependant, s'il est peut apparaître relativement "simple" de reconstituer l'ensemble de ses alliés pour une créature du laboratoire, la difficulté est autre lorsqu'il s'agit de mettre au point, hors du laboratoire, la traçabilité d'un parasite. En outre, cette tâche entre en tension avec la description du sociologue des sciences : en effet, la traçabilité est explicitement la recherche de l'ensemble des humains et non-humains qui ont permis l'existence de la chose tracée, mais, comme on l'a vu, en limitant le réseau à une série d'éléments pertinents. On peut dire que les acteurs étudiés par B. Latour et M. Callon cherchent à tenir leurs réseaux, au sens où ils doivent maintenir alignés tout une série d'éléments hétérogènes pour qu'il fonctionne à leur profit, alors que les nôtres visent à ce qu'ils soient bien tenus, c'est-à-dire que leur réseau puisse être ausculté, contrôlé, isolé, voire arrêté parce que son fonctionnement même peut être source de dangers.

De ce fait, le réseau n'est plus un descripteur largement extérieur aux acteurs mais l'enjeu même des luttes et controverses autour de la traçabilité : par exemple, doit-on tracer les Organismes Génétiquement Modifiés (OGM) dans des additifs largement diffusés dans les produits agro-alimentaires? Le faire, c'est mettre en place de nouveaux réseaux de

\footnotetext{
${ }^{14}$. Cf. notamment Latour (B.), Nous n'avons jamais été modernes, Paris, La Découverte, 1991.

15. Ainsi par exemple dans le cas de la production de piles à combustibles : 'L'électrode n'est pas seulement une réalité scientifique et technique ; elle n'est pas seulement une pièce maîtresse dans un programme politique ; elle est les deux à la fois. C'est une réalité composée. Elle constitue à elle toute seule un réseau socio-technique. Pour faire apparaître sa nature hybride ainsi que son extension, il a suffi de suivre le processus de sa construction, c'est-à-dire le recrutement de tous les alliés humains ou non humains qui ont dû être mobilisés pour lui donner sa forme en même temps que sa robustesse". Callon (M.), "L'agonie d'un laboratoire " in Callon (M.) (dir), La science et ses réseaux. Génèse et circulation des faits scientifiques, Paris, La Découverte, 1989, p. 189.
} 
surveillance. Y renoncer, c'est considérer la lécithine de soja comme "hors-réseau". La reprise en main par les acteurs de la notion de réseau ne nous oblige pas à l'abandonner, mais à prendre en compte le réseau qu'ils se donnent en renonçant aux descriptions totalisantes produites par le sociologue ${ }^{16}$. Si l'extension des réseaux est la cause première de l'innovation scientifique, nous en voyons en quelque sorte la face noire, la prolifération des parasites qui lui sont liés ${ }^{17}$. Et si une partie du travail d'attribution des découvertes/inventions ${ }^{18}$ consiste à effacer les traces passées des interventions des éléments du réseau hors laboratoire (interventions politiques, financières, ...), les acteurs chargés de la sécurité sanitaire vont au contraire se donner pour tâche de reconstituer un ensemble de traces fragiles du passage des parasites ${ }^{19}$.

\section{L'absence de mémoires externes face à des parasites discrets}

En l'absence des instruments associés à une politique de traçabilité - en particulier, la disposition de mémoires externes - la reconstitution des réseaux apparaît une entreprise des plus ardues. Circulant dans de vastes réseaux socio-techniques, certains parasites ont en effet pour propriété de demeurer très discrets ${ }^{20}$. Cela pose un problème quasi insurmontable pour les localiser dans le temps et l'espace. Bien sûr, lorsqu'ils se déplacent en quantités très importantes, comme à Tchernobyl, leur impact est immédiatement tangible. Mais contrairement à d'autres menaces plus habituelles, leur fréquentation n'a pas d'effets immédiats pour l'hôte, ce qui peut entraîner un diagnostic d'innocuité. C'est ce qui permet,

${ }^{16}$. Ce décalage peut être rapproché de celui effectué par Luc Boltanski à propos de la critique. Cf. Boltanski (L.), L'amour et la justice comme compétences, Paris, A.-M. Métailié, 1990.

${ }^{17}$. D'autres “faces noires” ont été décrites à partir du cadre du travail, Dodier (N.), Les Hommes et les Machines, Paris, A.-M. Métailié, 1996.

18. Expression que nous reprenons à I. Stengers, L'invention des sciences modernes, Paris, La Découverte, 1993. Pour une exemplification de ce dyptique au cas des instruments statistiques, voir Desrosières (A.), La politique des grands nombres, Paris, La Découverte, 1993.

${ }^{19}$. B. Latour illustre très précisément ce travail d'effacement effectué par les pastoriens parallèlement à leur propre reconstitution des réseaux de circulation des parasites (typhus, rage, diphtérie). Mais ce travail de reconstitution n'est qu'un moment du mouvement pastorien avant le passage au laboratoire et la fabrication éventuel d'un vaccin/sérum et non un enjeu de longue durée propre aux acteurs. Latour (B.), Les Microbes. Guerre et paix, Paris, Métailié, 1984.

${ }^{20}$ Cette discrétion est telle que l'on peut facilement mettre en cause leur dangerosité en l'absence de victimes avérées sur une longue durée. L'amiante contenue dans les flocages des bâtiments était ainsi souvent considérée comme inoffensive tant qu'elle demeurait confinée, innocuité d'autant plus soutenable que les cas de pathologies extra-professionnelles (hors des travailleurs de l'amiante et du bâtiment) n'étaient pas particulièrement apparents. Mais la poussière d'amiante, comme tout bon parasite, a des capacités de circulation (circuit d'aération...) peu prévues. Pour une description précise du dossier de l'amiante en France voir Chateauraynaud (F.), Torny (D.), Une analyse documentaire et sociologique du dossier de l'amiante (1970-1998), ronéo, 140p., septembre 1998. 
par exemple, à Roland Masse, alors président de l'Office de protection contre les rayonnements ionisants (OPRI) de relativiser le danger des faibles doses de radioactivité dans les aliments: "La France n'a édicté aucune limite de contamination pour les produits de consommation. Pourquoi ? Parce qu'en situation normale, ce n'est pas nécessaire. Alors c'est vrai qu'on peut trouver par-ci, par-là un crabe contenant 1250 becquerels. On peut même le manger vous savez! Ce n'est pas comme lorsqu'on trouve des salmonelles dans un fromage : là, l'impact sur la santé est immédiat" ${ }^{21}$. Comme on le voit, l'absence d'effets à court terme n'est pas sans conséquences du point de vue même de la reconstitution a posteriori des réseaux. Si d'autres effets sont supposés ou constatés à long terme, comment remonter aux causes premières de la contamination? En l'absence d'instruments liés à une politique de traçabilité, la seule solution consiste à solliciter la mémoire des acteurs eux-mêmes. La chose est difficile et source de nombreuses critiques. Ainsi à propos de l'enquête épidémiologique sur les leucémies menée par le professeur J.-F. Viel autour de l'usine de retraitement des déchets nucléaires de la Hague ${ }^{22}$, une des principales critiques fut celle du "biais d'anamnèse" : "Comment se souvenir combien de fois on est allé à la plage quand on était enceinte vingt ans plus tôt et d'où venaient les coquillages qu'on a mangés ?"23. Pareille critique peut apparaître de bon sens : en l'absence de mémoires externes, comment les personnes peuventelles reconstituer avec précision leurs déplacements et leurs actions sur des durées aussi longues que dix ou vingt ans? Malheureusement ce biais d'anamnèse n'est pas particulier à l'enquête du professeur Viel, mais porte sur presque toutes celles concernant l'effet des vecteurs de risques sanitaires, hôtes souvent si discrets qu'on les ignore ou les oublie facilement.

Le cas de la Maladie de Creutzfeldt-Jakob (MCJ) illustre parfaitement la difficulté à laquelle sont confrontés les agents de la sécurité sanitaire pour reconstituer des réseaux en l'absence de toute mémoire externe. Cette maladie peut en effet se déclarer jusqu'à quarante ans après un contact avec son agent contaminant, que nous dénommerons ici prion ${ }^{24}$. Lorsqu'un cas de MCJ est repéré, les acteurs du réseau de surveillance font remplir un questionnaire à un membre de la famille du patient, celui-ci n'étant en général plus en état d'y répondre luimême. Le questionnaire, utilisé au niveau européen, se présente sous la forme d'un cahier de quarante-quatre pages noircies de questions - ce qui est caractéristique d'une enquête tous

\footnotetext{
${ }^{21}$. Cité par Rivasi (M.) et Crié (H.), Ce Nucléaire qu’on nous cache, Paris, Albin Michel, 1998, p. 132.

${ }^{22}$. Pour une analyse détaillée de ce cas, voir dans ce même numéro, l'article de F. Chateauraynaud.

23. C. Hill, chercheuse à l'INSERM, citée par Rivasi (M.) et Crié (H.), Ce Nucléaire qu'on nous cache, op. cit., p.142. La citation provient de Libération du 10 janvier 1997.

${ }^{24}$. Techniquement le prion n'est qu'une hypothèse, certes solide, d'interprétation de la cause de la Maladie de Creutzfeldt-Jakob, tout comme chez l'animal, de la tremblante du mouton ou de la maladie de la vache folle.
} 
azimuts, lié à l'absence d'hypothèse dominante sur les facteurs de risque. "Le problème de ces questionnaires, nous confiait un membre du réseau, c'est que c'est assez pêche à la ligne ". En effet, la liste des questions inclut des facteurs d'identification classiques (sexe, âge, lieu de naissance, profession), des facteurs cliniques (date des premiers symptômes, nature des symptômes, date de diagnostic clinique) ou encore, des facteurs d'histoire médicale (opérations, transfusion, don du sang, greffes mais également traumatisme crânien, fréquentation d'un ophtalmologiste, maladies infectieuses, vaccinations, tatouages, utilisation de substances toxiques type tabac ou alcool, ...). Une autre partie du questionnaire inclut la famille du patient au sens élargi du terme, en prenant en compte l'âge actuel ou l'âge de la mort et le diagnostic de troubles nerveux. D'autres facteurs exogènes, plus spécifiques aux maladies à prions, sont également envisagés : 1'exposition aux animaux sous diverses formes (la viande, le cuir, le bétail, les morsures), avec spécification de ces animaux (chats, chiens, cochons, chevaux, moutons, bœufs, furets, belettes, animaux à fourrure, hamster, daim). L'alimentation, enfin, fait l'objet d'un traitement spécifique, lui aussi extrêmement détaillé. "Le questionnaire est fait en deux parties, à cause de la date, c'est-à-dire qu'on interroge sur les habitudes alimentaires avant 85 et après 85 ". Au final, les tableaux synthétiques utilisés donnent une idée des très grands efforts de mémorisation que les enquêteurs exigent de l'entourage du patient :

\begin{tabular}{|c|c|c|c|c|c|c|c|c|}
\hline Viande/Poisson & jamais & $\begin{array}{l}<1 \text { fois/ } \\
\text { an }\end{array}$ & $\begin{array}{l}\text { plusieurs } \\
\text { fois/an }\end{array}$ & $\begin{array}{l}>1 \text { fois/ } \\
\text { mois }\end{array}$ & $\begin{array}{l}>1 \text { fois/ } \\
\text { semaine }\end{array}$ & $\begin{array}{l}>1 \text { fois/ } \\
\text { jour }\end{array}$ & inconnu & incertain \\
\hline Agneau/Mouton & & & & $\mathrm{X}$ & & & & \\
\hline Porc/Jambon & & & & & $\mathrm{X}$ & & & \\
\hline Bœuf & & & & & $\mathrm{X}$ & & & \\
\hline Veau & & & & $X$ & & & & \\
\hline Gibier & & & & & & & $X$ & \\
\hline Volaille & & & & & & & & $X$ \\
\hline Poisson & & & & & $\mathrm{X}$ & & & \\
\hline Fruits de mer & $X$ & & & & & & & \\
\hline
\end{tabular}

Le questionnaire est en fait plus exigeant encore : pour chaque produit (par exemple les tripes), une distribution de fréquence est également proposée au répondant, ainsi qu'une demande de composition (ici des tripes). Pour le moment, il semble que cette enquête ait donné peu de résultats en termes de facteurs de risques liés à la MCJ. On voit ici l'ampleur de la tâche de reconstitution qui est nécessaire du fait de la grande variété des hôtes connus ou supposés pour le prion et de la durée potentielle de sa "période muette" au sein d'un organisme. Ainsi, même les réponses les plus sophistiquées en termes épidémiologiques semblent démunies face à des vecteurs comme les prions : il est très délicat, très coûteux et 
très incertain de retracer a posteriori l'ensemble des éléments ayant été au contact avec eux, dès lors que les expositions ne sont pas massives avec des effets tangibles sur une courte période. Face à cette impossibilité de reconstituer le passé, la traçabilité est une technique de gouvernement des personnes et des choses qui va contraindre par différents moyens la création d'une mémoire ordonnée pour le futur.

\section{La traçabilité : un modèle difficile à mettre en œuvre}

Rapport Mattei sur la "vache folle", Rapport Huriet sur la sécurité sanitaire, Rapport Deneux sur la qualité sanitaire des denrées destinées à l'alimentation humaine ou animale, Rapport Le Dréaut sur les OGM, projet de loi sur la création des ISS (Instituts de Sécurité Sanitaire) : la mise en place d'une sécurité sanitaire par la traçabilité est aujourd'hui largement discutée sur le plan réglementaire. Mais en quoi consiste précisément cette désormais fameuse traçabilité ? Il s'agit d'une notion dont on peut dire d'abord qu'elle recouvre un spectre de mesures très larges : la qualification des matières premières qui composent les produits (ainsi, les pouvoirs publics peuvent décider qu'un hamburger ne peut contenir tel ou tel tissu, qu'un flocage ne peut contenir de l'amiante...) ; la détermination des liens induits par la fabrication la circulation et l'utilisation (les pouvoirs publics peuvent exiger de connaître pour chaque produit l'ensemble des entités avec lesquels il est entré en contact, en définissant la nature du contact. Cette identification se fera souvent par lots, plutôt qu'individuellement, mais elle peut être individuelle. Ainsi, on peut exiger de connaître l'ensemble des patients ayant été en contact avec un instrument chirurgical donné, sur une période de temps donnée) ; l'étiquetage et le marquage des produits (chaque produit peut être identifié par un certain nombre de caractéristiques prédéfinies soit marquées sur le produit lui-même, soit, le plus souvent, attachées à son emballage. On peut ainsi indiquer sa provenance, son numéro d'identification, sa date de validité mais aussi s'il contient ou non OGM) ; la localisation des produits (dans le réseau de circulation, on peut à chaque instant savoir où se trouve un produit donné, ce qui peut permettre d'édicter une consigne, un retrait ou une destruction de produits lorsqu'ils sont soupçonnés d'être dangereux. Ainsi, les produits sanguins dérivés issus d'un donneur atteint ultérieurement de Maladie de Creutzfeldt-Jakob peuvent être immédiatement localisés) ; la conservation d'un échantillon de produits (les pouvoirs publics peuvent demander la conservation pour expertise d'une petite partie du produit ou d'un représentant d'un lot à des fins d'expertise pour déterminer par exemple la présence d'OGM).

Il s'agit en somme d'un ensemble de techniques qui vise à permettre à tout instant, par des procédés appropriés de constitution de mémoires externes aux personnes, la relocalisation des produits et des marchandises, sans pour autant enfreindre le principe de leur circulation. Les difficultés pratiques soulevées par cette modalité inédite de gouvernement des personnes et 
des choses sont nombreuses. Nous en examinerons quatre : la question des modes de sélection des populations concernées ; celle des modalités d'arrêt de l'action publique ; celle des compétences spécifiques attendues des citoyens ; celle, enfin, du "secret d'Etat" et de la transparence de l'information due au public.

Trier, éliminer, exclure : Quel est le bon mode de sélection des populations?

Un des premiers problèmes pratiques que rencontrent les décideurs et les experts qui ont en charge la mise en place des dispositifs de traçabilité est de savoir qui doit y être inclus et qui doit en être exclu. Cette question ne va pas de soi, contrairement à ce qui se passait dans le modèle du quadrillage et de la quarantaine. La délimitation des populations à suivre n'étant plus, ou étant moins directement liée à un lieu précis, à un territoire ou à une appartenance nationale, il s'agit en effet désormais de déterminer des critères qui permettront de distinguer préventivement qui fait partie du problème (c'est-à-dire : est vecteur potentiel de risques) ou qui n'en fait pas partie - à moins de considérer "tout le monde" sur un territoire donné comme vecteur potentiel, ce qui conduit précisément à un retour, toujours envisageable, vers le modèle de la quarantaine.

Les produits sanguins sont un bon exemple des problèmes de sélection des populations concernées que doit affronter le nouveau modèle. En France, le sang a été le siège d'un précédent dévastateur et particulièrement marquant, la contamination des transfusés et des hémophiles par le VIH, qui a fortement structuré le domaine, en particulier avec la création de l'Agence Française du Sang (AFS ${ }^{25}$. La sécurité transfusionnelle mise en place après l'affaire dite du sang contaminé s'est accompagnée d'un système d'hémovigilance assez sophistiqué, impliquant une traçabilité des produits. A l'intérieur de ce système, les premières mesures de prévention contre la transmission de la MCJ sont liées aux victimes du traitement du nanisme hypophysaire par hormone de croissance ${ }^{26}$. La sélection des donneurs apparaît comme la première mesure disponible pour lutter contre une éventuelle transmission d'éléments contaminants: toutes les personnes traitées à l'hormone de croissance doivent être considérées comme potentiellement à risque et écartées du don. Il s'agit selon l'AFS d'“une contre-indication absolue" ${ }^{\prime 27}$. Ce critère d'exclusion, cependant, ne permet pas en lui-même de

\footnotetext{
${ }^{25}$. D'autres problèmes se sont posés à la transfusion dont la massive contamination par l'Hépatite $\mathrm{C}$, mais celleci n'a pas eu jusqu'à présent le même effet visible de redéfinition des missions, structures et procédures de la transfusion sanguine.

${ }^{26}$. Rappelons que plus de cent enfants dans le monde, dont une majorité en France, sont décédés de MCJ suite à un traitement par hormone de croissance qui visait à contrer leur nanisme d'origine hypophysaire. Pour une analyse, voir Chateauraynaud et alii, op.cit., pp.240-251.

27. Agence Française du Sang, 23 décembre 1992.
} 
mettre un frein à toutes les inquiétudes. L'affaire du sang contaminé a montré le fossé entre les recommandations et leur application pratique, ainsi que les moyens de contrôle dont disposent les autorités régulatrices ${ }^{28}$.

Pour s'assurer de la validité du contrôle, les experts envisagent de jouer sur deux moyens différents : d'une part le questionnaire précédent le don de sang ne cesse d'être renforcé ; d'autre part la liste des personnes traitées par hormone de croissance est transmise depuis France-Hypophyse ${ }^{29}$ vers l'AFS. Deux techniques de repérage et de sélection des populations sont donc déployées conjointement : d'une part la participation active des donneurs qui portent avec eux leurs antécédents ; d'autre part des rapprochements effectués par des institutions sans passer par les donneurs. Chaque procédure a ses limites : dans le premier cas, elle requiert en chaque point du réseau de récolte une bonne volonté à la fois des personnels et des donneurs, en plus d'une capacité mémorielle de ces derniers. Dans le second, outre la nécessité d'un "service des recoupements" efficace, les personnes sont représentées par des données fixes (nom, adresse, date de naissance) dont certaines peuvent changer au cours du temps (mariage, déménagement). La mise en place de ces dispositifs lourds et susceptibles d'imperfections s'accompagne d'une multiplication des critères d'exclusion des donneurs. En application du "principe de précaution", la liste des contre-indications ne cesse de s'allonger :

L'interrogatoire médical pré-don doit permettre de détecter et d'écarter du don de sang, par mesure de précaution, les personnes susceptibles de présenter un risque de transmission de MCJ. A ce titre, sont considérées comme des contre-indications au don de sang : les traitements par hormone de croissance extractive d'origine humaine (actuellement l'hormone de croissance utilisée en France est issue du génie génétique). La notion d'antécédents de nanisme hypophysaire ainsi traité doit être recherchée ; les antécédents familiaux au 1er degré de MCJ: il conviendra dans ce cas de préciser les éléments sur lesquels le diagnostic a été porté ; les antécédents de greffe de tissus: l'entretien médical devra rechercher une greffe de cornée, une greffe de dure-mère pour réparation d'une brèche crânienne (des lambeaux de dure-mère ont pu être également utilisés dans le cadre d'interventions articulaires mais le sujet l'ignore le plus souvent) ; les antécédents d'intervention neurochirurgicale ou d'exploration cérébrale invasive (examen stéréotaxique) à cause de l'utilisation possible d'instruments susceptibles d'avoir été employés chez des sujets atteints de $\mathrm{MCJ}^{30}$.

\footnotetext{
28. Rappelons que la mesure d'exclusion du don du sang des "groupes à risques", dont les homosexuels à partenaires multiples, définie par la circulaire "Soulier" DGS/3B N 569 du 20 juin 1983, avait provoqué une polémique publique importante, et, de ce fait, eu une application très faible. Voir Libération, "Les pédés, groupe sanguin indésirable ?”,16 Juin 1983 et Strazzulla (J.), Le Sida 1981-1985, Paris, La documentation française, 1994. Sur l'importance de ce document pour l'affaire du sang contaminé, voir Hermitte (M.-A.), Le Sang et le Droit, Paris, Seuil, 1996.
}

\footnotetext{
${ }^{29}$. Il s'agit d'une association à laquelle l'Etat a confié la gestion de l'approvisionnement et de la distribution de l'hormone de croissance. Ses responsables ont été mis en examen suite à différents rapports administratifs et aux plaintes de parents d'enfants malades ou décédés.

${ }^{30}$. Agence Française du Sang, 24 mai 1995.
} 
Le développement de l'attention portée aux prions créé un contexte favorable à la remontée et à la prise en compte de vieilles inquiétudes jusque là absente du champ des préoccupations. Ainsi par exemple, vingt ans après les premières descriptions de son possible pouvoir contaminant, la greffe de cornée entre dans les critères d'exclusion. Cet allongement des critères d'exclusion soulève deux problèmes distincts: la mise en application du questionnaire pré-don devient de plus en plus fastidieuse avec un risque de baisse d'attention de la part des protagonistes, et la multiplication des exclusions peut faire surgir à terme un problème d'approvisionnement. L'aboutissement de cette logique se trouve dans l'exclusion annoncée en décembre 1996 de l'ensemble des receveurs de produits labiles, ceux-ci étant considérés comme plus "à risque" en raison de leur absence de traitement inactivant (chauffage,...), contrairement aux produits dérivés. Mais est-ce encore suffisant ? Ne faut-il pas pousser plus loin les principes d'exclusion ? L'annonce publique, en mars 1997, d'une expérimentation apportant des éléments tangibles sur la transmission du prion par le sang suscite des positions plus radicales ${ }^{31}$ : "Ne faut-il pas écarter du don, même si la disposition peut être source d'incompréhensions, tous les donneurs ayant fait l'objet d'un traitement par produits sanguins, dans la mesure où un doute plane ? Le bénévolat du don et le caractère non lucratif de la collecte du sang, s'ils soulignent la noblesse du système transfusionnel français, n'en garantissent pas, à eux seuls, la qualité et la fiabilité du système sanitaire" ${ }^{\prime 32}$. Comme pour d'autres activités, l'adoption de mesures de précaution de plus en plus draconiennes face aux alertes peut tendre à terme vers une cessation ou du moins, à une réduction très significative de l'activité même de la transfusion sanguine par manque de donneurs. Dans ce cas, le modèle de la traçabilité touche une limite, puisqu'il ne permet plus la poursuite de la production et de l'échange des produits.

\section{Ne pas faire du monde un laboratoire :Quelles modalités d'arrêt pour l'action publique?}

Un second problème pratique lié à la mise en place des dispositifs de traçabilité est de savoir comment arrêter l'action publique une fois qu'elle s'est lancée dans la remontée aux origines des produits et des personnes. La chaîne de contrôle, dont les implications économiques peuvent être lourdes, doit-elle être indéfiniment étendue dans des cas où le risque n'est pas absolument avéré ? Ce problème se pose par exemple dans le cas des dispositifs médicaux implantables qui recouvrent quantité de produits différents: valves cardiaques, lentilles

\footnotetext{
${ }^{31}$ Les conditions de cette parole publique sont problématiques : Paul Brown, expert reconnu des prions, a donné un entretien au Sunday Times qui a mis en avant les résultats d'une expérience encore en cours, dont les résultats n'étaient en conséquence ni définitifs, ni même susceptibles de communication scientifique. Suite à cet article, Paul Brown fut invité à une conférence de l'Organisation Mondiale de la Santé (OMS), qui publia un communiqué ambigu, entraînant une importante couverture médiatique, voir par exemple Le Monde du 27 Mars 1997.
}

\footnotetext{
32. L'Humanité du 1er avril 1997.
} 
intraoculaires, colle chirurgicale, catgut, greffes osseuses, fragments cutanés, cornées, souvent issus du monde animal. Dans ce domaine, il existe depuis avril 1994 un Groupe d'experts sur la sécurité microbiologique des dispositifs médicaux, produits et procédés utilisés à des fins médicales. A l'été 1997, celui-ci avait étudié trois cent trente-six dossiers. Composé d'experts de différents domaines, son rôle est d'autoriser et de définir les conditions d'utilisation de tous les dispositifs médicaux implantables. Sa grande originalité est de définir une liste positive de ces dispositifs, c'est-à-dire que tous les produits qui ne sont pas sur cette liste sont interdits ${ }^{33}$.

Même si les prions ne sont pas les seules menaces pour la sécurité sanitaire, les conditions nécessaires pour leur élimination sont les plus draconiennes. Pour autoriser un produit, le comité demande à ce que le fabricant lui fournisse un ensemble de pièces concernant tous les types de contrôle liés à la traçabilité avec des conditions particulières concernant la sécurité microbienne, bactériologique, virale ou prions, ce qui représente le plus souvent plusieurs centaines de pages. Si ces documents ne satisfont pas les experts, le dossier est placé en sursis à statuer, en attendant la fourniture de nouveaux documents, la clarification du dossier ou, plus rarement, le changement des procédures de fabrication (inversion d'étapes par exemple). $\mathrm{Au}$ troisième sursis à statuer, le dossier est refusé par le Comité. En revanche, si le dossier est conforme, un avis favorable est émis. Cet avis n'est pas définitif, il se situe dans une dynamique des connaissances autour du prion ou de ses hôtes. Chaque produit est individualisé. Ainsi le même produit fabriqué par la filiale $\mathrm{X}$ du groupe Sorip, autorisé précédemment, nécessite une nouvelle procédure s'il est produit par la filiale $\mathrm{Y}$, parce que l'origine biologique des produits n'est pas la même. Néanmoins, nos acteurs n'étant pas ethnométhodologues, tout n'est pas renégocié à chaque fois : le Comité s'appuie lui-même sur d'autres formes de certifications (Autorisation de Mise sur la Marché ou Avis du Comité de sécurité virale de l'Agence du Médicament) pour étayer son jugement.

Pour illustrer les difficultés d'arrêt de l'activité de contrôle que rencontre le Groupe d'experts, prenons l'exemple d'un adjuvant utilisé en chirurgie ophtalmique, dont le composant principal est du hyaluronate de sodium, lui même extrait de crêtes de coq. Il s'agit à la fois de connaître les conditions de fabrication d'un tel produit, du point de vue de la sécurité virale, microbienne et bactérienne. La question de l'utilisation et d'une éventuelle alternative thérapeutique sont évoquées. Mais c'est la qualification de l'origine des produits qui est centrale. En effet, la régression vers l'origine peut être infinie et ce sont les conditions de l'arrêt qui sont au cœur du processus de contrôle. Tout d'abord, les coqs ne sont pas euxmêmes soupçonnés d'héberger des prions. Néanmoins, ils ingèrent des aliments sous forme de

\footnotetext{
${ }^{33}$. D'après un responsable de la DGS, cela est contraire au droit européen dans son principe de libre circulation.
} 
farines. Le fabricant du hyaluronate de sodium affirme, documents à l'appui, qu'il ne s'agit pas de farines de mammifères, ce qui laisse la place aux poissons ou aux volailles. Des alertes ont circulé sur les saumons d'élevage, fondées sur la découverte de protéine PrP (sous une forme normale) dans leur encéphale. Faut-il alors également exiger de contrôler l'origine des farines ? Faut-il également surveiller la nourriture des poissons qui sont à l'origine de cette farine ? Le comité en a-t-il le pouvoir réglementaire ? En revanche, en aval, les conditions de contrôle sont bien définies avec une traçabilité liée à chaque lot.

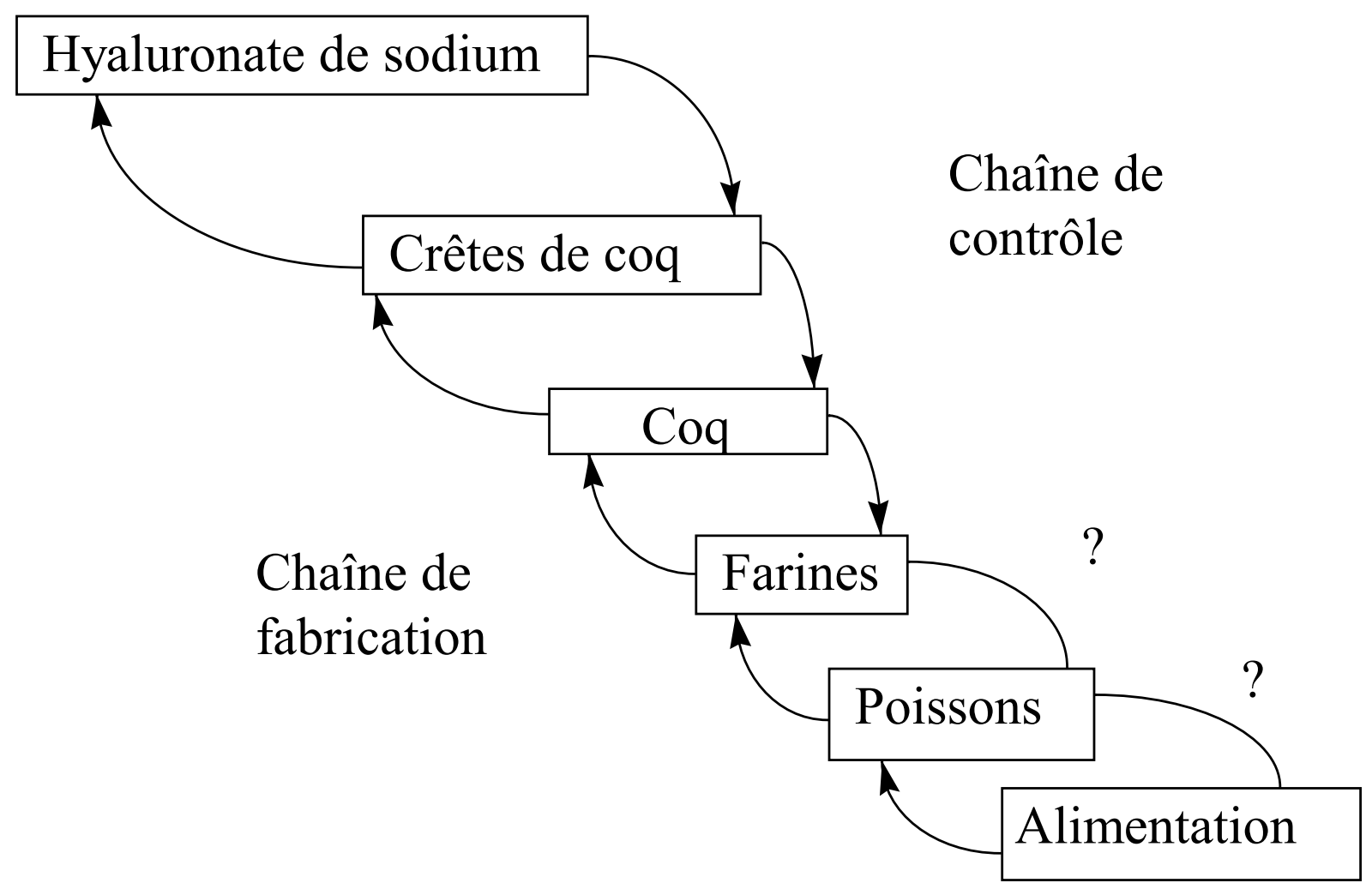

Ce dispositif montre clairement les problèmes liés au contrôle et à l'application pratique du "principe de précaution". Où s'arrêter dans une chaîne de traçabilité ? Où la commencer ? Où la finir ? Ne peut-on considérer les prions comme susceptibles d'envahir d'autres supports, aujourd'hui inimaginables? En contraignant une très forte traçabilité avant même l'installation de tout dispositif de surveillance, et a fortiori, dans la plupart des cas, de toute victime, le Groupe d'experts adopte, par application du "principe de précaution", un mode de fonctionnement liés aux matières dangereuses. En cela, il pousse loin l'activité de contrôle, en subordonnant la question du coût économique à l'impératif de sécurité. Reste que la chaîne, à un moment donné, doit être arrêtée, à moins de transformer l'ensemble du monde en laboratoire. Arrêt qui reste problématique et peut toujours être soupçonné d'être arbitraire ou dangereux. 
Quelles compétences sont attendues des citoyens?

Un troisième problème pratique lié à la mise en place des dispositifs de traçabilité est pour les décideurs et les experts de déterminer le rôle qui revient aux citoyens dans leur mise en œuvre. Ce rôle, comme nous allons le voir, est appelé à être plus actif et plus important qu'il ne l'était dans le modèle précédent de la quarantaine. Le modèle de la traçabilité engage en effet une nouvelle définition de la vigilance citoyenne. Considérons par exemple le cas de la crise de la "vache folle" de mars 1996. Suite à cette crise, l'étiquetage Viande Bovine Française (VBF) a imposé la traçabilité sur l'ensemble de la filière, "de la fourche à la fourchette" ou, autre slogan, "de l'étable à l'étal", afin de garantir au consommateur une viande provenant d'un animal né, élevé et abattu en France. Antérieurement à ces mesures, deux systèmes de traçabilité différents existaient déjà : d'une part les bovins vivants étaient suivis via leur numéro d'identification, qui individualisait chaque animal auquel était associé un certain nombre de documents. D'autre part, les carcasses étaient identifiées et cette identification demeurait présente après découpe, ou du moins était disponible si aucun paquetage ne permettait un marquage (par exemple chez les détaillants bouchers). Quelles étaient les conséquences de cette séparation entre animal vivant et viande ? L'abattoir était la plaque tournante où les animaux, quelque part entre la salle d'abattage et la salle de découpe, perdaient toute identification, ce qui rendait impossible par la suite la remontée vers l'élevage en cas de problème du côté de la filière aval, que ce soit pour appliquer des sanctions ou pour marquer une qualité d'élevage particulière ${ }^{34}$. Evidemment, en cas de problème sanitaire non repéré par les structures compétentes, et qui provenait des conditions d'élevage, il était impossible d'opérer un rappel de produits ou d'interdire la circulation de certains produits.

Le nouveau dispositif de traçabilité, étendu sur l'intégralité de la filière, peut être désormais utilisé pour traiter y compris des menaces anciennes - par exemple, un problème sanitaire classique en élevage : l'utilisation d'hormones aujourd'hui illégales, dont la visibilité a été particulièrement forte à la fin des années 70 avec l'affaire du veau aux hormones. En 1996, en Bretagne, un détaillant boucher, au moment de préparer sa viande, aperçoit des cicatrices d'injection dans des cuisseaux de veau. Il pense à des injections d'hormones ("parce que normalement on ne fait pas d'injections dans le cuisseau, parce que c'est le morceau le plus noble. C'est dans le collet qu'on injecte" nous l'explique le vétérinaire qui a suivi l'affaire). Il précise :

\footnotetext{
${ }^{34}$. La qualité des carcasses est évaluée à l'abattoir par des agents spécialisés, résultant d'un jugement expert et non de marques partagés par tous (type de nourriture, type d'élevage...). Sur l'importance de la perception dans l'expertise voir Bessy (C.), Chateauraynaud (F.), Experts et faussaire, Paris, A.-M. Métailié, 1995.
} 
Le boucher a remonté l'information ici [à l'abattoir] et on a pu remonter jusqu'au veau, jusqu'à l'éleveur, donc là il y a eu une traçabilité parfaite du détaillant. Là avertissement à l'éleveur et je communique le nom de l'éleveur à la coopérative et à la brigade d'enquête vétérinaire qui s'occupe de démanteler les filières d'anabolisants.

Dans un cas comme celui-ci, la traçabilité nécessite d'abord une attention particulière, ici celle d'un détaillant boucher, pour percevoir des traces suspectes. Il est vraisemblable qu'un simple consommateur n'aurait pu les percevoir, ou du moins, n'aurait pu les interpréter en termes de traces d'injections. Mais le boucher aurait aussi pu se contenter de découper sa viande et de la vendre, en supposant que le consommateur est précisément moins bien équipé pour repérer ce type de traces. Tout au contraire, il met en forme son soupçon et fait passer l'alerte à l'abattoir, doté de nouveaux moyens informatiques permettant d'assurer cette traçabilité, de sanctionner l'éleveur et d'activer une autorité de tutelle, la Brigade Nationale d'Enquêtes Vétérinaire (BNEV), qui pourrait prendre des mesures pour contenir ces "veaux aux hormones" hors de la consommation humaine

Le modèle de la traçabilité n'exige pas seulement cette nécessaire vigilance citoyenne de la part des différents intervenants de la chaîne de commercialisation. Pour fonctionner, il réclame également que ces intervenants s'informent sur les procédures de fabrication et de transformation des produits qu'ils diffusent. Ainsi par exemple, dans le cas des Viandes Séparés Mécaniquement (VSM), de nombreuses mesures ont été prises pour interdire certaines parties des bovins dans ces "viandes" qui entrent ensuite dans la composition de nombreux produits industriels (raviolis, tartes, hamburgers). L'arrêté du 23 avril 1996 interdit notamment l'utilisation des têtes, pieds, moelle épinière, colonne vertébrale issus des bovins nés avant le 31 Juillet 1991. De façon plus générale, ce sont les tissus considérés comme les plus infectants (classe I et II de l'OMS) qui sont éliminés de la consommation humaine. Mais comment s'assurer que d'autres tissus n'entrent pas dans la consommation ? Un vétérinaire, dans l'ouest de la France, a été amené à s'interroger sur cette question. Il s'était documenté sur les méthodes d'abattage en Grande-Bretagne, découvrant qu'il peut s'y pratiquer par pistolet pneumatique - méthode susceptible de provoquer la migration de tissus cervicaux vers les artères pulmonaires ${ }^{35}$. Il connaissait par ailleurs les effets des traumatismes crâniens en médecine humaine, provoquant des embolies. Il a fait le rapprochement avec les conditions d'abattage des bovins et a donc cherché des tissus cervicaux dans d'autres organes. Or, les poumons (classe IIIb) ne font l'objet d'aucune mesure particulière et sont utilisés dans la fabrication de certaines saucisses. Finalement, la mise à jour, par une expérimentation vérificatrice, d'un tel lien entre tissus de classe I et produits de consommation humaine a pu entraîner de nouvelles mesures de contrôle (interdiction de fabrication, changement de technique d'abattage). Cet exemple illustre le fait que si la traçabilité des viandes n'est pas

\footnotetext{
${ }^{35}$. Cf. "Brain emboli in the lungs of cattle after stunning", The Lancet, vol . 348, 31 août 1996.
} 
aujourd'hui directement utile en terme de santé publique par rapport à la Maladie de Creutzfeldt-Jakob, son absence dans le cas des abats ou des VSM rend pensable l'introduction de tissus potentiellement dangereux dans la chaîne alimentaire. Ce n'est que par un travail attentif d'attention et de vérification, composantes complémentaires de la vigilance, que la traçabilité peut remplir son rôle ${ }^{36}$.

Marquer, immatriculer, étiqueter : comment peut-on informer le public?

Jusqu'à présent, nous avons mis en scène la traçabilité dans un cadre de filière professionnelle ou d'expertise scientifique et technique. La vigilance citoyenne attendue était celle des différents intervenants dans la chaîne de production, de commercialisation et de contrôle sanitaire. Or, la participation des profanes, du "grand public", est l'une des contraintes majeures du bon fonctionnement de la traçabilité en "monde ouvert", sous peine d'y perdre une bonne partie de son efficacité. C'est ce que suggère l'exemple de cet élève, qui déjeunant à la cantine de son collège, a trouvé dans son steack une aiguille d'injection, de la taille de celles qui servent dans les soins donnés aux bovins. Dans ce cas, les vétérinaires ont pu "remonter" jusqu'à un certain nombre d'éleveurs. "Parce que, comme l'explique l'un d'eux, c'était vraiment un collège assez important donc ils avaient acheté énormément de sous-vide, et ils avaient bien sûr gardé les étiquettes, mais ils ne savaient plus de quel paquet le steak provenait, comme ils en avaient un grand nombre. Par contre avec toutes ces étiquettes, on a pu remonter jusqu'à huit éleveurs, environ dix bovins. Il y a dix bovins qui ont pu donner ce steak avec une aiguille dedans. Alors, on a bien sûr fait remonter l'information jusqu'aux coopératives et sur l'abattoir, les éleveurs, mais on n'a pas pu mettre de procès-verbal, puisqu'on ne savait pas lequel des huit c'était". Comme le montre cette anecdote, les contraintes deviennent extrêmement importantes lorsqu'on quitte un espace professionnel pour aller vers un monde plus ouvert, en particulier des espaces collectifs. D'où un quatrième problème pratique lié à la mise en place des dispositifs de traçabilité : comment assurer le contrôle jusque dans l'assiette afin que l'alerte qui résulterait d'un consommateur puisse aboutir quelque part ? Comment informer le public pour qu'il participe à l'action de vigilance attendue de lui ?

La traçabilité des viandes peut être utile dans le cadre de certains incidents sanitaires, mais cela demande une tangibilité importante du phénomène: l'aiguille est immédiatement perceptible, le prion, lui, ne l'est pas. Vu les délais de contamination, relier les cas de MCJ à une consommation particulière est pratiquement impossible, à moins que chacun ne conserve toutes les identifications de l'ensemble de ces consommations sur dix ou quinze ans, et encore

\footnotetext{
${ }^{36}$. Sur une construction théorique de la vigilance voir Chateauraynaud (F.), "Vigilance et transformation", Réseaux, n85, sept-oct 1997, pp. 101-127.
} 
faudrait-il pouvoir ensuite recouper les informations. Ce qui paraissait déjà extrêmement présomptueux en terme d'interrogation avec le questionnaire du réseau de surveillance se transforme ici en entreprise paranoïaque. Néanmoins, on peut, dans un premier temps, s'assurer d'une traçabilité des supports ayant le plus d'affinités avec les agents pathogènes : un certain nombre d'abats sont interdits pour cette raison. La Direction Générale de l'Alimentation (DGAL) cherche à inclure dans les modes d'exclusion de ces abats une coloration résistant à de nombreux traitements afin de permettre leur identification en cas de réintroduction dans la chaîne alimentaire. Là encore, la traçabilité implique d'abord de rendre tangibles les propriétés des "viandes" elles-mêmes. Le consommateur ne peut en effet s'appuyer sur ses capacités sensorielles pour déterminer la présence ou l'absence de nombreux parasites tels les OGM : "La difficulté est que l'utilisation du génie génétique est relativement inodore et sans saveur dans de nombreux produits" ${ }^{37}$. La solution consiste alors à lui fournir sous une forme claire un étiquetage qui lui indique des éléments issus de la traçabilité ${ }^{38}$.

Dans le domaine des OGM, cette question des modes et conditions d'étiquetage se trouve au centre de la controverse. Les experts du Conseil National de l'Alimentation, prenant en compte la nécessité de laisser au consommateur lui-même le choix de promouvoir ou non l'utilisation des nouvelles technologies génétiques, envisagent en effet un étiquetage qui lui permette de faire la part entre produits avec ou sans OGM :

\begin{abstract}
Dès lors qu'il s'agit de denrée composée, aucun ingrédient, quelle que soit sa teneur, ne devra être issu d'OGM. Les termes à utiliser pour cette allégation sont : "produit obtenu sans recours aux techniques de modification génétique". Une mention du type "animal nourri avec des produits obtenus sans recours aux techniques de modification génétique" pourrait aussi être employée pour des denrées d'origine animale, à condition que cette allégation puisse être démontrée. L'utilisation de toute référence à une mention négative ne doit être possible que si la preuve de l'allégation peut être établie sans ambiguïté. Seule une traçabilité sans faille permet d'apporter une garantie fiable de cette mention. Des démarches de contractualisation des opérateurs d'une filière, du producteur jusqu'au transformateur, sont nécessaires pour l'établissement de cette traçabilité. Un système de certification, qui s'inscrirait dans les dispositions existantes (agriculture biologique, certification de conformité, label) assurerait une transparence et donc une crédibilité à ces allégations ${ }^{39}$.
\end{abstract}

L'étiquetage est considéré ici comme un devoir d'information vis-à-vis du consommateur afin de lui rendre l'initiative dans le choix des produits avec ou sans OGM. Par rapport à la

\footnotetext{
${ }^{37}$ Intervention de J.-F Molle, directeur général, chargé du département " Sécurité alimentaire, réglementation et environnement " du groupe Danone, L'utilisation des organismes génétiquement modifiés dans l'agriculture et dans l'alimentation, Rapport 545, Office Parlementaire d'évaluation des Choix Scientifiques et Techniques (OPCST)Tome 2, partie 1, 1998

${ }^{38}$. On connait de ce point de vue l'importance de l'apparition du logo VBF dans la limitation de la baisse de consommation des produits bovins après la crise de mars 1996.

${ }^{39}$. Avis du Conseil National de l'Alimentation (CNA) sur l'étiquetage "sans OGM ”,17 Juin 1997.
} 
traçabilité, l'étiquetage représente une extension au-delà des filières professionnelles : là où le nucléaire ou l'aéronautique créaient un système à destinations des professionnels, le domaine alimentaire va au-delà en introduisant le consommateur dans la chaîne de traçabilité. Mais celui-ci se trouve dans une position de dépendance vis-à-vis des filières professionnelles, qui sont seules capables d'assurer l'adéquation entre l'étiquetage et l'absence d'OGM. Pour introduire cette traçabilité, deux méthodes différentes sont envisagées : celle fondée sur l'origine des ingrédients utilisés (qui implique une traçabilité des matières incorporées) et celle de l'analyse au cas par cas des lots d'ingrédients utilisés (qui implique une détection systématique $)^{40}$. Les formes de preuve et d'attestation de l'absence de vecteurs de risques potentiels sont diverses et ne coïncident pas nécessairement: passer par le laboratoire ne garantit que des qualités de l'échantillon et du lot qui lui est éventuellement lié alors que la traçabilité seule s'appuie sur la certitude qu'à chaque moment (semences, transport, transformation) le réseau de circulation est parfaitement défini et "étanche". Ce n'est qu'en combinant les deux (mais à quelle fréquence, à quel coût ?) qu'on s'assure de la validité de la traçabilité par la visibilisation, en laboratoire, de l'absence ou de la présence des OGM. Le consommateur, informé par la restitution de la tangibilité via l'étiquetage peut toujours basculer dans l'inquiétude à moins de se doter lui-même d'un laboratoire portable.

\section{Du consommateur au receveur de produits sanguins : jusqu'où faut-il informer le public?}

L'information du consommateur est l'un des buts explicites de la traçabilité des viandes, et plus généralement des denrées alimentaires. Mais qu'en est-il du patient qui n'a pas fait un choix de consommation mais a reçu par nécessité des produits sanguins ${ }^{41}$. Faut-il eux aussi les prévenir de la présence éventuelle, et même fortement hypothétique, de vecteurs de risques mortels? Le texte évoqué plus haut à propos de la sélection des donneurs envisageait déjà le problème des receveurs, en déployant un dispositif de mémoires externes propre à l'institution et mobilisable à l'avenir :

Les informations relatives aux receveurs éventuels devront être conservées selon une procédure définie par l'E.T.S. [Etablissement de Transfusion Sanguine], de manière à pouvoir retrouver rapidement ces receveurs si des éléments nouveaux apparaissaient, notamment au niveau des risques de transmission et des possibilités de diagnostic en fonction de l'évolution des connaissances $^{42}$.

\footnotetext{
${ }^{40}$. Ibid.

41. Cette nécessité est néanmoins discutable non dans l'absolu mais en terme de quantités et d'équilibre bénéfice/risque. On sait que le VIH a provoqué une forte diminution de l'utilisation des transfusions dites de confort. Il est possible que le prion ait le même type d'effet.

${ }^{42}$. Il s'agit ici des receveurs de produits labiles.
} 
Conserver une information sur un risque éventuel sans prévenir les personnes concernées peut être problématique et peut se révéler décisif dans le passage à l'affaire comme dans le cas des contaminations par hormone de croissance ${ }^{43}$. Pour résoudre cette question, le Ministre de la Santé s'est tout d'abord adressé au Comité d'Ethique (CCNE). Celui-ci a répondu en concluant négativement sur le passage de l'alerte et la jugeant infondée :

Le Comité National d'éthique estime qu'en l'état actuel des connaissances, il n'existe aucune argument en faveur de la possibilité de contamination par le sang, bien qu'évidemment la preuve absolue de l'innocuité ne puisse être donnée. Informer les receveurs qu'ils courent, ou feraient courir, par don de leur sang, un risque infime contre lequel on ne peut rien ne paraît pas éthiquement acceptable. Les écarter du don de sang sans qu'ils en soient informés ne semble pas davantage possible ${ }^{44}$.

Deux questions différentes sont ici abordées : d'une part une alerte concernant les receveurs eux-mêmes, d'autre part une alerte dérivée où le sang des receveurs devient lui-même un support créant de nouveaux liens par le don du sang. Le second problème a été tranché par le Ministère en excluant du don tout receveur de produits labiles. Mais, comme pour les autres systèmes de contrôle, encore faut-il que celui-ci en soit informé, à moins bien sûr de conserver l'ensemble des traces des produits reçus, ce qui peut être problématique, en particulier dans le cadre hospitalier.

"Avez-vous reçu des produits sanguins ?" : comment répondre à cette question si on n'a pas été spécifiquement informé ? De plus, il faut être capable de reconnaitre ces produits lorsqu'on nous les administre. Prenons l'exemple des immunoglobulines antitétaniques. Celles-ci sont administrées systématiquement à un patient qui est admis dans une structure hospitalière avec une plaie ouverte, afin d'éviter une contamination par le tétanos. Si l'on veut s'assurer demain que ces patients sont bien conscients d'avoir reçu ces produits, il n'existe aucune structure de représentation qui permettent de les atteindre : "Nous ne nous adressons pas à 1500 ou 2000 hémophiles parfaitement identifiés, mais peut-être à 50000 ou 70000 malades qui circulent en France dont on connaît pourtant le nom. Les pharmaciens qui pratiquent la traçabilité disposent de listes qui dorment dans leurs ordinateurs !" ${ }^{45}$. Le

\footnotetext{
43. La question de l'information du patient est largement discutée de façon plus générale, en particulier avec l'installation de la norme du "consentement éclairé". Mais les prions rendent encore plus délicate l'information, y compris dans le cas du traitement par hormone de croissance: "c'est au médecin traitant qui connaît le patient et sa famille d'apprécier quand et comment il doit informer s'il peut le faire. Logique pour toute personne concernée qui souhaite connaître les risques, les symptômes et l'évolution d'une telle maladie. Abusif, peut-être, si l'on impose une vérité douloureuse à qui ne veut ou ne peut l'entendre. (...) Libre au [médecin] de délivrer directement une information, somme toute très difficile car un certain mystère demeure face à cette affection, ou encore d'adresser son patient à un spécialiste", "Risque de transmission : une vigilance mesurée ",Panorama du médecin, 3 octobre 1994.

44. Avis du CCNE, avril 1996.

${ }^{45}$. Chast (F.), “Concilier le doute et l'efficacité”, Conférence à l'Espace Ethique, 18 juin 1997.
} 
problème reste donc entier. C'est pourquoi, suite à une nouvelle alerte liée aux produits sanguins, le Ministère demandera un nouvel avis au Comité d'Ethique et à l'Académie de Médecine, laquelle répondra par cette formule : "Toute personne a droit à une information loyale, claire et approprié sur son état. Dans tous les cas [1'Académie recommande] une information large, précise et continue du corps médical". Même si cette déclaration a entraîné de nombreux débats sur la lourdeur de la gestion des procédures d'information et sur l'angoisse qu'on pourrait créer inutilement chez les receveurs, l'évolution qu'elle marque est le signe d'une mise en place d'une nouvelle politique de la vigilance, incluant ici des citoyens sans qualité particulière (tout le monde peut avoir reçu du sang) dans un dispositif de surveillance et de contrôle.

Une telle politique de concernement des citoyens n'est pas sans opposition. En effet, le CCNE a lui aussi répondu au ministre, réponse plus tardive (1er octobre 1997). Pour sa part, le Comité sépare nettement l'information du médecin et l'information du malade. Dans le premier cas, l'intérêt de santé publique et de recherche est central : "L'identification des malades ayant reçu ce type de produits doit être dès maintenant prospectivement enregistrée afin de permettre des études épidémiologiques ultérieures et de tracer facilement les malades en cas de nécessité" ${ }^{46}$. Mais ces nécessités sont extérieures à la personne du malade : celui-ci n'a pas d'action dans les dispositifs de recherche et de traçabilité. En conséquence, il n'y a pas nécessité de l'informer en l'absence de certitudes. L'alerte est ici réduite à un générateur d'inquiétude qui ne peut trouver de point d'appui pour se résorber :

L'information sur le risque potentiel que comportent certaines thérapeutiques est d'une autre nature que l'information sur le traitement lui-même quand elle ne repose pas sur des faits scientifiquement établis. Dès lors qu'un risque est connu, scientifiquement démontré, l'information du malade s'impose. Si le risque est virtuel, théorique, cette information n'a pas de justification éthique car elle peut être ressentie comme une menace inconnue, diffuse, qui peut inciter à des comportements irrationnels dangereux pour le malade lui-même et pour la société. C'est actuellement le cas pour $[\mathrm{la} \mathrm{MCJ}]^{47}$.

Ce qui se joue dans cette controverse entre CCNE et Académie de Médecine, c'est bien l'extension de la politique de traçabilité : doit-on la confiner dans un espace professionnel délimité ou l'étendre dans un espace public a priori illimité ? Ou dans un autre domaine, à quoi sert-il d'informer sur les plus petits incidents du parc nucléaire, si ce n'est créer l'inquiétude? Les experts sont-ils les seuls qui peuvent déterminer la pertinence de telles informations et agir en conséquence ? Le modèle de la traçabilité conduit en définitive à choisir entre deux anthropologies politiques : soit on dote les personnes de capacités de

\footnotetext{
${ }^{46}$. CCNE, “Avis sur l'information à donner aux patients à propos de la possibilité de transmission de l'agent de la maladie de Creutzfeldt-Jakob par des composants du sang ”, n55, 1er octobre 1997.

47. Ibid.
} 
vigilance propres, utiles à elles-mêmes mais également partageables, d'un sens normal de l'inquiétude et on les inclut éventuellement dans des dispositifs de traçabilité. Soit on considère que, face à une incertitude, elles peuvent adopter des comportements "irrationnels dangereux" pour elles-mêmes et "pour la société". A cela s'ajoute la question de la durée : dans le premier cas, les personnes sont dotées de capacités de circulation, ce qui rend difficile leur suivi à partir d'un dispositif panoptique, dans le second cas, on peut, "facilement", à chaque inflexion scientifique du risque, les tracer.

Dans la lutte contre la dissémination des parasites en "monde ouvert", la traçabilité, telle que nous l'avons décrite ici, a essentiellement deux fonctions. D'une part, elle permet d'introduire ce qu'on pourrait appeler des "barrières" à l'entrée des réseaux, barrières qui visent, par l'adoption de procédures de tri et d'exclusion, à prévenir l'introduction de menaces potentielles. D'autre part, parce qu'il est aujourd'hui largement admis par les acteurs chargés de la sécurité sanitaire que ces barrières ne suffisent pas et ne suffiront jamais, la traçabilité doit permettre de doter les pouvoirs publics d'une capacité de rattrapage des produits en circulation (retrait, destruction, consigne, information) liée à une possibilité de repérage d'événements surgissant en un point donné du réseau mis sous surveillance.

Tout comme l'organisation de la "discipline" au sens de Foucault s'étendait, pour atteindre son but, bien au-delà de ses institutions les plus visibles (en devenant un véritable modèle d'organisation socio-politique), la traçabilité, pour réussir à contenir l'émergence multiple de menaces, tend aujourd'hui à être étendue au-delà des univers professionnels où elle est déjà implantée. De ce fait, la renégociation des frontières entre univers professionnels et profanes constitue, comme nous l'avons vu, un premier enjeu qu'introduit le nouveau modèle. Ce n'est pas le seul: la circulation des parasites, liée à celle des personnes et des marchandises, dépasse largement le cadre de l'Etat-Nation qui était présupposé dans les exemples précédents. Il y a là une difficulté supplémentaire qu'illustre bien le cas des OGM :

J'ai en tête l'affaire de Kochko dans le Tarn-et-Garonne. Je la résume brièvement car nous l'avons reçu dans nos auditions. Il s'agit d'un agriculteur biologique du Tarn-et-Garonne qui fait du soja biologique, avec un cahier des charges. Il vendait son soja à un exportateur de Poitiers, qui l'a vendu en Allemagne. Lors d'un contrôle en Allemagne par les techniques PCR (...) on a découvert que le tofu fabriqué à partir de son soja avait une "contamination" non-prévue d'un soja génétiquement modifié. On remonte la filière et on arrive chez l'agriculteur. Le problème de la responsabilité se pose. Lorsqu'il s'adresse au semencier, celui-ci dit que c'est exempt d'OGM. On a fabriqué ces semences aux Etats-Unis. Lors de la fabrication des semences, certains pollens sont vraisemblablement venus de champs de sojas génétiquement modifiés. La contamination était sans 
doute faible, mais nous allons arriver à la même chose au niveau de notre pays, et cela posera le problème de l'étiquetage et de la traçabilitét ${ }^{48}$.

La dimension internationale de la circulation des parasites potentiellement vecteurs de dangers sanitaires pose la question du cadre juridique pertinent lors d'une éventuelle recherche de responsabilité, elle-même indexée sur la capacité variable de mise en jeu de la traçabilité. Une solution consisterait à confiner les réseaux de circulation de certains types de produits à l'intérieur de l'espace national. Pour cela encore faudrait-il s'assurer de l'absence de parasites déjà présents sur le territoire : on sait que ce fut le choix du Centre National de la Transfusion Sanguine (CNTS) en interdisant l'importation de produits sanguins et en maintenant une collecte et une fabrication de produits non chauffés au niveau national ${ }^{49}$. En l'absence d'un tel choix "protectionniste", la pratique intensive du "poolage" qui démultiplie les liens entre donneurs et receveurs rend rapidement indispensable une forte traçabilité (dont l'acceptation nécessite des accords et certifications supranationales) pour pouvoir recouper les informations nécessaires à un éventuel retrait rapide des lots.

Contrairement aux mesures de quadrillage et d'isolement, les mesures fondées sur la traçabilité n'ont pas pour "moteur" la certitude d'un danger. Ces mesures s'appuient simplement sur des indices, des éléments de preuve suffisamment tangibles, et sont donc toujours susceptibles de fortes critiques - dont une forme paradigmatique est "l'absence de risque zéro". Comme nous l'avons vu, ces mesures ont par vocation un aspect tâtonnant et évolutif, les populations concernées et les dimensions des chaînes de contrôle étant susceptibles de se modifier rapidement - par exemple, sous l'effet de mises en évidence scientifiques du caractère contaminant d'un vecteur jusqu'alors non pris en compte. En définitive, c'est dans un entre-deux entre la certitude et le soupçon permanent lié à un fonctionnement paranoïaque - garder en mémoire l'ensemble des éléments du monde et leur contacts et transformer la planète entière en laboratoire ${ }^{50}$ - que se situent les formes pratiques de la traçabilité.

En France, en raison des précédents de l'affaire du sang contaminé et de la "vache folle", c'est la sécurité sanitaire qui s'affirme aujourd'hui comme le lieu privilégié et le champ d'expérimentation d'une traçabilité exigeante, débordant des seules filières professionnelles pour concerner aussi les profanes. En tant que modèle de gouvernement des choses et des

\footnotetext{
${ }^{48}$. J.-Y. Le Dréaut, Député, Président de l'OPCST, L'utilisation des organismes génétiquement modifiés dans l'agriculture et dans l'alimentation, op.cit. Tome 2, partie 1

${ }^{49}$. Cf. Hermitte (M.-A.), Le sang et le droit, op.cit.

50. Sur le mode de fonctionnement de la recherche comme paranoïaque voir Latour (B.), La vie de laboratoire, Paris, La Découverte, 1989.
} 
personnes, la traçabilité est cependant susceptible de s'étendre bien au delà du seul domaine des risques collectifs. A bien des égards, elle se présente comme un nouveau moyen tout à la fois de développement et de régulation du capitalisme. D'une part, la traçabilité est utilisée par des organisations critiques du néolibéralisme afin de mettre en jeu des conditions de circulation des produits et services : absence de travail des enfants, rémunération minimale des employés, droit de grève doivent être garantis par les producteurs non seulement dans les limites de leur entreprise, mais à travers l'ensemble de leur chaîne de sous-traitance, sous peine de boycotts ${ }^{51}$. D'autre part, la traçabilité est affichée par ces mêmes producteurs comme une garantie de sécurité par des retraits immédiats de produits ciblés, mais aussi de qualité, de fiabilité et de service : c'est parce qu'ils contrôlent l'ensemble de la chaîne de production qu'ils peuvent garantir " 1 'authenticité " (c'est à dire l'origine, le mode de production ou de transformation), "l'éthique " (c'est-à-dire l'absence de recours au travail des prisonniers ou des enfants pour la fabrication des marchandises) ou l'assurance d'un paiement équitable aux producteurs de matière première. Comme nous avons essayé de le montrer, la principale conséquence de la généralisation de ce nouveau modèle sera, au plan socio-politique, une redéfinition en profondeur de la relation que l'Etat et les citoyens sont amenés à entretenir.

\footnotetext{
${ }^{51}$. Les polémiques autour du fabricant d'articles de sport Nike forment un paradigme de ces discussions.
} 